\title{
Políticas de indexação na América Latina
}

\author{
Indexing policies in Latin America
}

\author{
Mariângela Spotti Lopes FUJITA (1) e Isidoro GIL LEIVA (2)
}

(1) Universidade Estadual Paulista Júlio de Mesquita Filho - UNESP, Av. Hygino Muzzi Filho, 737, Campus Universitário, CEP. 17525900 - Marília, SP, Brasil, Caixa-Postal: 421, fujita@marilia.unesp.br; (2) Universidad de Murcia, Facultad de Comunicación y Documentación, 30100 Campus de Espinardo, Espinardo (Murcia), España, isgil@um.es

\begin{abstract}
Resumen
La política de indización no debe ser vista como una lista de procedimientos a seguir, sino como una filosofía que refleje los intereses y objetivos de una unidad de información. Eso nos lleva a pensar sobre la indización desde el punto de vista gerencial y estratégico en el contexto de unidades de información. Una política de indización básica debería contemplar la forma de realizar la indización, los vocabularios controlados y de listas de autoridades (identificadores, nombres de personas, nombres de lugares, etc.) manejados, así como los mecanismos de revisión y evaluación de la indización. Con el objetivo de llevar a cabo un estudio sobre las políticas de indización latinoamericana en Bibliotecas Nacionales y Archivos Nacionales, así como de algunos sistemas de información, se realizó un diagnóstico exploratorio y descriptivo compuesto de una investigación teórica y otra práctica a través del funcionamiento y los procedimientos de indización desde la perspectiva de la gerencia y del indizador, y la evaluación de acceso y la recuperación de la información por el usuario. Para obtener una cobertura representativa de América Latina, en primer lugar, fueron seleccionados los países más importantes de la región y, posteriormente, se realizaron las visitas presenciales desde octubre de 2008 hasta junio de 2009. En los datos hasta ahora recopilados, se observa una clara inexistencia de políticas de indización en las veinticuatro instituciones visitadas.
\end{abstract}

Palabras clave: Política de indización. Indización. Bibliotecas Nacionales. Archivos Nacionales. Sistemas de información.

\section{Introdução}

Com a finalidade de fornecer um panorama latino-americano da política de indexação de Bibliotecas Nacionais, Arquivos Nacionais e principais sistemas de informação, realizou-se estudo diagnóstico exploratório e descritivo composto de investigação teórica e pesquisa de campo (1).

A proposta da pesquisa "Política de indexação latino-americana" decorreu do desenvolvimento da pesquisa "Política de tratamento da informa-

\begin{abstract}
The indexing policy must not be considered as a list of procedures to be followed, but a philosophy that reflects the interests and aims of the information unit. This takes us to think about indexing from the managerial and strategic point of view in the context of information unities, as it happens in the input and output of the information system. A basic indexing policy should consider the indexing methodologies, the use of controlled vocabularies and lists of authorities (identifiers, author names, place names, etc.), as well as assessment mechanisms. With the purpose of developing a study on the policy of the Latin American indexing in National Libraries and National Archives, as well as of some information systems, it was carried out an exploratory and descriptive diagnosis that consisted of two parts: the functioning and the procedures of indexing in the perspective of management and of the indexer, and the evaluation of information access and retrieval by the user. In order to obtain a representative range of Latin America, the most important countries of South America and Central America and Caribbean were selected in two blocks. With the data analysis collected up to now, it was possible to observe that the results show the inexistence of indexing policies in the twenty-four visited institutions.
\end{abstract}

Keywords: Indexing policy. Indexing. National libraries. National archives. Information systems.

ção documentária na rede de bibliotecas da UNESP" (2) que realizou um estudo diagnóstico para coleta de dados com uma amostra de nove bibliotecas universitárias do sistema de bibliotecas da UNESP em três áreas do conhecimento - Odontologia, Engenharia Civil e Pedagogia. Os resultados, além de revelarem elementos específicos da política de indexação no contexto de bibliotecas universitárias, propiciaram uma experiência de abordagem e estratégia metodológica quanto à pesquisa de campo para a coleta de dados com os informantes nas instituições 
selecionadas e alertaram para a necessidade de investigar outras unidades de informação em localizações geográficas diferentes, a fim de obtermos mais dados para estudos comparados no que tange à indexação como tratamento temático da informação documentária em bibliotecas, arquivos e sistemas de informação especializados em áreas do conhecimento.

A indexação é um processo de análise documentária realizado sobre os documentos com a finalidade de determinar-lhes um conjunto de palavras chave ou assuntos para facilitar sua armazenagem em bases de dados e sua posterior recuperação para atender necessidades de informação. Até o aparecimento da Internet cada instituição realizava a indexação de seus documentos pensando unicamente em seus próprios usuários da instituição. A colocação na Internet dos catálogos e dos documentos digitalizados de importantes instituições documentais (Bibliotecas Nacionais, Arquivos Nacionais ou grandes serviços de indexação e resumos) está propiciando que os cidadãos em geral, e os investigadores em particular, possam satisfazer suas necessidades de informação sem deslocar-se fisicamente até onde estão os documentos. Daí que as políticas de indexação deveriam contemplar de alguma maneira este novo contexto sem perder de vista seus usuários mais próximos.

A maioria das grandes instituições documentais deveria contar com uma política de indexação explícita com o objetivo de alcançar a máxima coerência possível nas tarefas de representação da informação. Uma política de indexação básica deveria contemplar a forma de realizar a indexação, o uso de vocabulários controlados e de listas de autoridades (identificadores, nomes de pessoas, nomes de lugares, etc.), assim como mecanismos de revisão e avaliação.

Com esta perspectiva, o estudo compõe-se de estudo diagnóstico de funcionamento e procedimentos de indexação na perspectiva da gerência e do indexador em perspectiva latinoamericana; análise dos vocabulários controlados e as listas de autoridades (nomes próprios, lugares, etc.) empregadas na indexação e análise comparativa do gerenciamento de políticas de indexação entre bibliotecas nacionais, arquivos nacionais e sistemas de informação latinoamericanos.

\section{Pressupostos teóricos}

O processo contínuo de informatização dos serviços de tratamento da informação documentária permitiu que sistemas de recuperação da informação (índices, catálogos, inventários), antes locais e restritos à determinada comunidade, são agora disponíveis pela Internet, atravessando fronteiras físicas e temporais. No nosso ponto de vista, essa visibilidade dos sistemas de recuperação da informação faz com que o profissional da informação assuma uma nova responsabilidade compromissada com a realidade não somente de sua comunidade usuária local, mas também de uma comunidade usuária potencial virtual cada vez mais exigente.

Nesse contexto, é importante afirmar que a visibilidade desses sistemas de recuperação da informação e a responsabilidade do profissional da informação permeiam todo o processo de tratamento de forma e conteúdo, tornando-se necessária a adoção de condutas profissionais com base na definição das diretrizes de uma política de indexação que resulte no aprimoramento e consistência do processo de tratamento para obtenção de qualidade de recuperação da informação.

Essa política torna-se importante porque visa à gestão da informação registrada de modo a dar visibilidade na recuperação da informação para as bibliotecas e serviços de indexação e resumos, além de identificar condutas teóricas e práticas das equipes de tratamento da informação documentária envolvidas para definir um padrão de cultura organizacional coerente com a demanda da comunidade acadêmica interna e externa.

A política de indexação não deve ser vista como uma lista de procedimentos a serem seguidos, e sim uma filosofia que reflita os interesses e objetivos da unidade de informação. Isso nos leva a pensar sobre a indexação do ponto de vista gerencial e estratégico no contexto de unidades de informação, uma vez que ela acontece na entrada e na saída de informações do sistema, confirmando-se como (Fujita, 1999).

a parte mais importante porque condiciona os resultados de uma estratégia de busca, produzindo uma correspondência precisa com o assunto pesquisado em índices.

A indexação, e por conseguinte sua política, deve fazer parte do planejamento dos sistemas de informação de modo a compor-se como um conjunto de procedimentos, materiais, normas e técnicas orientadas por decisões que refletem a prática e princípios teóricos da cultura organizacional.

De acordo com Carneiro (1985, p. 221) uma política de indexação

deve servir como um guia para tomada de decisões deve levar em conta os seguintes fatores: características e objetivos da organização, determi- 
nantes do tipo de serviço a ser oferecido; identificação dos usuários, para atendimento de suas necessidades de informação e recursos humanos, materiais e financeiros, que delimitam o funcionamento de um sistema de recuperação de informações.

Por outro lado, segundo Gil Leiva (2008, p. 297):

Por política de indexação se entende tanto a forma de realizar a indexação de uma determinada instituição como o esforço por concretizar, sistematizar e representar em manuais os processos seguidos na indexação. Esta representação dos procedimentos em manuais deve estar em relação direta com o número de profissionais que se dedicam a esta tarefa ou o grau de qualidade perseguido pelo sistema.

A política de indexação engloba, assim, todos os procedimentos, materiais, normas e técnicas de tratamento de conteúdo documentário.

Com relação às perspectivas de uma política de indexação em Bibliotecas Nacionais a literatura mais recente demonstra interesse, sobretudo no que se refere ao acesso temático das Bibliografias Nacionais produzidas, e apresenta alguns estudos diagnósticos desenvolvidos em alguns países da Europa e da América do Norte com subsídios para estudos comparados que poderemos aproveitar quanto aos países da América Latina.

O grupo de trabalho sobre Diretrizes para Acesso por Assunto das Agências Bibliográficas Nacionais (Working Group on Guidelines for Subject Access by National Agencies) considera que (Bourdon; Landry, 2007, p. 2, tradução nossa):

o desenvolvimento de uma política de indexação/catalogação (é) um passo necessário para oferecer aos usuários uma informação adequada sobre o tipo disponível de acesso por assunto para os documentos listados em bibliografias nacionais. O objetivo do grupo é fazer recomendações às bibliotecas nacionais sobre os tipos de documentos que serão indexados de acordo com as necessidades de vários grupos de usuários.

Bourdon e Landry (2007) ressaltam que devem ser discutidos elementos-chave para determinar a relevância e utilidade da política de indexação para os diferentes grupos de usuários:

- Política de indexação: aspecto estratégico: Para quem a política é direcionada? Quais os limites de sua aplicação? A política deveria ser publicada? Deveria incluir o histórico da política?

- Apresentação das ferramentas para indexação: Quais as diferentes linguagens e esquemas de classificação utilizados? Quais as ca- racterísticas de cada linguagem? A indexação será pré ou pós-coordenada?

- Aplicação das ferramentas para indexação: Como as ferramentas serão utilizadas na bibliografia nacional e no catálogo da biblioteca nas agências bibliográficas nacionais? Existirão níveis diferentes de catalogação? Devem ser levados em conta os projetos de indexação retrospectiva? Quais os tipos de documentos indexados e não indexados? O índice será bilíngüe / multilíngüe? Qual o uso das ferramentas para indexação na interface de busca? As ferramentas serão disponibilizadas para os usuários durante suas buscas?

Observa-se, que há uma volta à teoria da indexação/catalogação de assunto e dos estudos ressaltando a importância da elaboração de sua política não mais somente direcionada aos grandes sistemas de informação especializados, mas também às bibliotecas nacionais.

Sobre indexação na perspectiva de sistemas de informação especializados, Fattahi (1998) apresenta interessante ponto de vista sobre a tendência de integração entre as bases de dados bibliográficas e os serviços de indexação e resumos dentro do contexto dos catálogos on-line. Essa aproximação faz com que se abra uma janela para todo o corpus bibliográfico, fazendo com que esses serviços sejam fatores de destaque para o catálogo. Nesse cenário, o usuário espera maior consistência dentro e entre os dois sistemas.

A introdução de novas tecnologias, conforme Fattahi (1998) fez com que a catalogação nas bibliotecas e os serviços de indexação tivessem um maior impacto um sobre o outro devido aos seguintes fatores:

- Integração e acessibilidade de diferentes catálogos on-line e serviços de indexação e resumos através de um único terminal;

- A capacidade de busca e a recuperação eficaz, que a tecnologia introduziu aos catálogos de biblioteca, são copiadas dos serviços de indexação e resumos, indicando o impacto desses serviços nas práticas de catalogação;

- A inclusão e a indexação de um amplo espectro de publicações, tais como dissertações, anais de congressos, relatos de pesquisa, pelos serviços de indexação e resumos têm influenciado os princípios e as regras que são usados para criação de bases de dados;

- Evolução das versões de CD-ROM e bases de dados que agora são disponibilizadas aos usuários. 
Rubi (2000; 2004) observa, que em sistemas de informação especializados, tais como International Nuclear Information System (INIS), International Information System for Agricultural Sciences and Technology (AGRIS) e Centro LatinoAmericano e do Caribe de Informação em Ciências da Saúde (BIREME), existe o profissional indexador, que pode ser um bibliotecário (AGRIS E BIREME) ou um especialista (INIS) na área de atuação do sistema.

Esses sistemas de informação internacionais funcionam como catalisadores e disseminadores de informações. Formados por um conjunto de centros referenciais de informação especializada interdependentes com objetivos comuns, apresentam como características: âmbito informacional definido; informação concentrada; pesquisas especializadas; fontes primárias e secundárias; reduto de especialistas; conexões com outros centros e sistemas de informação nacionais e internacionais. (RUBI, 2000).

Com a colaboração dos centros referenciais, esses sistemas de informação são responsáveis pela produção, comercialização e distribuição de produtos e serviços relacionados à informação especializada como bases de dados; bibliografias especializadas; disseminação seletiva da informação; boletins periódicos, dentre outros.

A produção de bases de dados faz com que seja necessária a utilização do procedimento de indexação, realizado por profissionais altamente qualificados, bibliotecários ou especialistas da área, e utilizando como ferramentas os manuais de indexação especialmente elaborados e linguagens documentárias específicas como os tesauros.

No Brasil, são referências de sistemas de informação especializados: o Centro LatinoAmericano e do Caribe de Informação em Ciências da Saúde (BIREME), o Centro de Informações Nucleares da Comissão Nacional de Energia Nuclear (CIN/CNEN) que integra o International Nuclear Information System (INIS), o Centro Nacional de Agricultura (CENAGRI) que integra o International Information System for Agricultural Sciences and Technology (AGRIS) e o Centro de Documentação do Senado Federal (PRODASEN). Com certeza, existem outros sistemas, mas os citados têm política de indexação bem definida e são reconhecidos pela comunidade científica da área de Ciência da Informação no Brasil e devem servir como parâmetro para o estudo exploratório desta pesquisa.

Em uma busca na Internet para identificação de sistemas de informação especializados em países da América Latina nos deparamos com a falta de uma sistematização e de um quadro pouco definido e desequilibrado com relação à existência de sistemas de informação especializados em diferentes áreas.

Em contato feito por correio eletrônico com alguns profissionais e pesquisadores em Ciência da Informação de países da América Latina, obtivemos algumas considerações interessantes acerca desse panorama. A Professora Adjunta, Maria Cristina Perez, Coordenadora Docente da Escola Universitária de Biblioteconomia do Uruguai considerou a pesquisa muito importante e necessária, haja vista que no Uruguai são poucos os sistemas de informação produtores de bases de dados especializadas e que somente poucas áreas de assuntos participam de programas cooperativos bibliográficos nacionais $\mathrm{e}$ regionais, salvo os setores que considera "forte" como o sistema de informação biomédica que participa da BIREME e desenvolve uma rede de bibliotecas biomédicas em nível nacional. Nesse sentido, espera-se que o estudo exploratório a ser realizado durante as viagens de coleta de dados para a pesquisa possa identificar alguns sistemas de informação especializados em países latino-americanos.

$\mathrm{Na}$ Espanha encontramos profissionais dedicados principalmente a realizar tarefas de indexação somente no IEDCYT (Instituto de Estudios Documentales sobre Ciencia y Tecnología, antes chamado CINDOC), mais especificamente na alimentação das bases de dados produzidas pelo Conselho Superior de Investigações Científicas (Bases de dados ICYT, ISOC e IME). Por outro lado, em geral, os arquivos na Espanha não dão muita importância à indexação, mas desde uns anos atrás estão começando a utilizar massivamente a norma ISAD (g) para a descrição de documentos de arquivo e estão colocando os catálogos na internet com a determinação de descritores temáticos e de locais geográficos, principalmente. Do mesmo modo, existe pouca literatura sobre política de indexação em arquivos e, também, é recente o uso de tesauros em arquivos. O mesmo panorama pode ser visto em termos de política de indexação para arquivos no Brasil. Dessa forma, uma vez coletados os dados sobre indexação nos Arquivos Nacionais latinos americanos pretende-se realizar um estudo comparado entre arquivos da Espanha e do Brasil.

\section{Metodologia}

As bases da investigação teórica e o planejamento da pesquisa de campo foram desenvolvidos em Marília, com a colaboração da $\operatorname{Prof}^{a} \operatorname{Dr}^{a}$ Mariângela Spotti Lopes Fujita, e a pesquisa de 
campo para a coleta de dados e documentação foi desenvolvida a partir de Marília pelo Prof. Isidoro Gil Leiva em visitas aos seguintes países latino-americanos: Argentina, Uruguai, Paraguai, Chile, Peru, Equador, Colômbia, Panamá e Costa Rica.

Esta pesquisa iniciou-se, no Brasil, na Universidade Estadual Paulista (Marília, São Paulo), onde se concretizou, definitivamente, a investigação com a Prof ${ }^{\mathrm{a}} \mathrm{Dr}^{\mathrm{a}}$ Mariângela Spotti Lopes Fujita, considerando-se a experiência de planejamento, coleta de dados, análises e resultados obtidos durante o desenvolvimento do Projeto "Política de tratamento da informação documentária na rede de bibliotecas da UNESP".

O desenvolvimento da pesquisa de campo constou de coletas de dados por entrevistas, aplicação de questionários e consultas à documentação de Bibliotecas Nacionais, Arquivos Nacionais e Sistemas de Informação, com as seguintes etapas:

(a) Entrevistas com indexadores para análise de procedimentos de indexação;

(b) Entrevistas com os dirigentes das unidades de informação para conhecimento do planejamento global e da política de indexação, além de obtenção de documentos de serviço;

(c) Entrevistas com usuários para análise da recuperação da informação.

\begin{tabular}{|c|c|}
\hline Mês / Ano & Países \\
\hline \multicolumn{2}{|l|}{2008} \\
\hline Outubro & $\begin{array}{l}\text { Marília (Brasil). Planejamento } \\
\text { definitivo da pesquisa }\end{array}$ \\
\hline Novembro & $\begin{array}{l}\text { (América do Sul) } \\
\text { Argentina } \\
\text { Paraguai }\end{array}$ \\
\hline Dezembro & $\begin{array}{l}\text { Chile } \\
\text { Uruguai }\end{array}$ \\
\hline \multicolumn{2}{|l|}{2009} \\
\hline Janeiro & $\begin{array}{l}\text { Peru } \\
\text { Equador }\end{array}$ \\
\hline Fevereiro & Colômbia \\
\hline Março - Abril & $\begin{array}{l}\text { (América Central) } \\
\text { Panamá } \\
\text { Costa Rica }\end{array}$ \\
\hline Maio - Julho & $\begin{array}{l}\text { Volta ao Brasil (UNESP, Marília). } \\
\text { Análise dos dados coletados }\end{array}$ \\
\hline
\end{tabular}

Quadro 1: Orientação planificada da viagem.

Para realizar as entrevistas semi-estruturadas foi aplicado questionário (vide Apêndice $A$ ) nas unidades de informação selecionadas cuja ela- boração teve como objetivo verificar a existência ou não de um manual de procedimentos para a indexação, o uso de sistemas automáticos para realizar esta tarefa ou para a validação dos termos ou determinação de assuntos, as linguagens de indexação utilizadas, etc.

O cronograma do desenvolvimento da pesquisa e das visitas às instituições selecionadas se realizou do seguinte modo:

O planejamento inicial teve que ser modificado devido ao surto da "Gripe Suína" no México em meados de abril de 2009. Esta situação provocou o impedimento das visitas às instituições do México e de alguns países da América Central.

As instituições visitadas até final de maio de 2009 foram as seguintes:

- Argentina: Biblioteca Nacional, Arquivo Nacional.

- Uruguai: Biblioteca Nacional, Arquivo Nacional.

- Paraguai: Biblioteca Nacional, Arquivo Nacional e Centro de Documentación y Estudios.

- Chile: Biblioteca Nacional, Arquivo Nacional.

- Peru: Biblioteca Nacional, Arquivo Nacional e Centro Internacional de la Papa.

- Equador: Biblioteca Nacional, Arquivo Nacional e CONAMU.

- Colômbia: Biblioteca Nacional, Arquivo Nacional e Biblioteca Universidad de Manizales.

- Panamá: Biblioteca Nacional, Arquivo Nacional e Biblioteca Autoridad del Canal de Panamá.

- Costa Rica: Biblioteca Nacional, Arquivo Nacional e BINASSS.

\section{Resultados}

Como relatado anteriormente, em cada uma das vinte e quatro instituições visitadas foi preenchido presencialmente um questionário (Apêndice A), do qual vamos revisar abaixo as questões mais importantes.

\section{Manual de procedimentos para a indexação?}

Das vinte e quatro instituições visitadas somente quatro manifestaram dispor de um manual de procedimento para a indexação mesmo que nenhum tenha sido publicado.

2. Uso de algum sistema automático para realizar a indexação? 
Nenhuma das instituições visitadas usa sistemas para a atribuição automática ou semiautomática da indexação.

3. Seleção automática de assuntos ou descritores de listas de autoridades?

Somente quatorze instituições utilizam listas de autoridades durante a catalogação ou descrição de documentos dos quais selecionam aqueles assuntos ou descritores que desejam atribuir ao registro em questão. Apenas dois Arquivos nacionais, o Arquivo Nacional de Chile e o da Colômbia, empregam este sistema para a atribuição da indexação por meio de vocabulários construídos por eles mesmos.

4. Uso de termos de vocabulário natural, não controlado?

Das vinte e quatro instituições visitadas somente três usam vocabulário em linguagem natural em algum campo de suas bases de dados.

\section{Linguagens de indexação usadas?}

Das nove bibliotecas nacionais visitadas somente uma delas não determina assuntos nem descritores durante o processo de catalogação dos documentos porque não utilizam nenhum tipo de linguagem de indexação. As demais bibliotecas nacionais usam as seguintes linguagens de indexação (A ordem das bibliotecas é a que se realizaram as visitas e das linguagens de indexação, em que foram nomeadas):

-B. Nacional do Uruguai: LEM Carmen Rovira, 1967; LEM CSIC; LEM BU Sevilla; LEMB Luis Ángel Arango ; LCSH.

- B. Nacional do Paraguai: LEM Carmen Rovira, 1967; LEM Bcas públicas España; Tesauro de la UNESCO.

-B. Nacional do Chile: Library of Congress Subject of Headings (LCSH) traduzida; Lista de Cabeçalhos de Assuntos (LCA) própria.

- B. Nacional do Peru: LCSH desde 1994; Lista de Encabezamientos de Materia para Bibliotecas (LEMB) Luis Ángel Arango ; LEM CSIC ; Lista de autoridades de catálogos on line (Brasil, Espanha, França, etc.).

- B. Nacional do Equador: Arquivo de autoridades de assuntos em Formato MARC. Armarc para pequenas bibliotecas. Colombia, 2007; LEMB Luis Ángel Arango, 1985.

-B. Nacional da Colombia: LEMB Luis Ángel Arango; Tesauro de música.

- B. Nacional do Panamá: LEMB Luis Ángel Arango.
- B. Nacional da Costa Rica: LEMB Luis Ángel Arango.

Como se pode observar, seis destas bibliotecas usam, em maior ou menor medida, a Lista de Encabezamientos de Materia para Bibliotecas (LEMB), editada pela Biblioteca Luis Ángel Arango da Colombia.

Entretanto, nas bibliotecas especializadas ou centros de documentação visitados, o uso de linguagens de indexação é mais variado devido à própria natureza dessas instituições. Aqui aparece o uso de diferentes tesauros de acordo com a documentação tratada, mas também é utilizada a LEMB Luis Ángel Arango em duas delas (Biblioteca da Autoridad del Canal de Panamá e na Biblioteca da Universidad de Manizales, Colombia).

Com respeito aos Arquivos Nacionais visitados cabe ressaltar que o Arquivo Nacional do Chile dispõe de uma lista de assuntos composta por umas 1200 entradas para a indexação dos documentos notariais. O Arquivo Nacional do Peru conta com uma base de dados que contém a descrição da documentação notarial do século $\mathrm{XVI}$ com um campo denominado descritores de onde se incluem temas ("Daños y perjuicios") e onomásticos ("Yugular, José"), bem como realizam tarefas de normalização para nomes próprios, títulos nobiliários, cargos, etc. Por último, destaca-se que o Arquivo Nacional da Colômbia é o único Arquivo em que utilizam um software específico para a gestão de Arquivos. A base de dados está estruturada de acordo com a norma internacional de descrição ISAD (g) e conta com campos específicos para receber a indexação, tais como "Descritores onomásticos", "Descritores geográficos" e "Descritores temáticos". Com os descritores temáticos que possuem (uns 200.000 aproximadamente) estão trabalhando na construção de um tesauro.

6. Participa ou já participou de projetos de interoperabilidade entre vocabulários controlados?

Das vinte e quatro instituições visitadas somente uma delas (B. Nacional do Chile) participou, em finais de 1980, em um projeto de interoperabilidade de vocabulário controlado com outras instituições do país no contexto da criação de um catálogo coletivo.

7. Realizam uma avaliação periódica da indexação atribuída aos registros?

Nenhuma das instituições visitadas realiza uma avaliação periódica da indexação atribuída. Quase todas confirmaram que esta tarefa não pode ser realizada por falta de pessoal. 
8. A instituição dispõe de uma política de indexação?

Das vinte e quatro instituições visitadas somente uma delas (B. Nacional do Chile) respondeu que dispõe de uma política de indexação regulamentada apesar do documento não estar publicado.

\section{Considerações finais}

A execução deste projeto nos permitiu conhecer importantes instituições documentais da América Latina, tanto Bibliotecas Nacionais, Bibliotecas especializadas, Centros de Documentação e Arquivos nacionais. Como se depreende dos dados apresentados, a maior parte destas instituições não dispõe de uma política de indexação. Esta situação é devida a várias circunstâncias. Em uns casos porque algumas das instituições não realizam tarefas de indexação, aqui se enquadra a maioria dos Arquivos nacionais, em outros casos porque não se aperceberam da importância e repercussão direta que a sistematização e representação por escrito dos processos de indexação têm na qualidade dos sistemas de armazenamento e recuperação da informação. E em terceiro lugar, um aspecto importante que foi constantemente referido em quase todas as instituições: por falta de pessoal.

Por último, cabe destacar que este projeto nos abre várias linhas para que possamos seguir trabalhando. Por um lado, na sistematização teórica de políticas de indexação que, posteriormente, cada instituição possa adaptar a suas necessidades e realidade. E por outro lado, uma tarefa de maior alcance e mais ambiciosa relacionada com a interoperabilidade ou compatibilidade entre vocabulários controlados. A implantação e uso da internet de maneira generalizada e, em particular, o esforço que muitas instituições estão realizando em bibliotecas e coleções digitais, requer um trabalho paralelo e cooperativo entre os países, com o fim de desenvolver e oferecer ferramentas cada vez mais universais. Neste contexto de universalização da informação é necessário começar a dar os passos necessários para o desenvolvimento de uma linguagem de indexação online em espanhol e português que possa ser usada, ao menos, pelas Bibliotecas nacionais da Península Ibérica e da América Latina. Esta ferramenta seria o andaime básico para, em um futuro mais distante, conectar as bibliotecas digitais desenvolvidas por estas instituições por meio de um catálogo coletivo que contribuiria diretamente para a difusão e universalização do conhecimento disponível.

\section{Notas}

(1) Desenvolvido, com apoio do CNPq, em bases de cooperação científica entre a Profa. Dra. Mariângela Spotti Lopes Fujita e o Prof. Dr. Isidoro Gil Leiva da Universidade de Múrcia, Espanha que obteve bolsa Pesquisador Visitante Estrangeiro do CNPq para o período out.2008 a jul.2009.

(2) Coordenação de Mariângela Spotti Lopes Fujita Projeto vinculado ao Programa de Gestão 2005/2008 da Rede de Bibliotecas da UNESP.

\section{Referencias}

Bourdon, F., Landry, P. (2007) Best practices for subject access to national bibliographies: interim report by the Working Group on Guidelines for Subject Access by National Bibliographies Agencies. Durban: IFLA, 2007. http://www.ifla.org/IV/ifla73/papers/089-Bourdon_Landryen.pdf (21 ago. 2007).

Dias, M. do R. I. (1999) Catalogação e qualidade: breve estudo. São Paulo: UNESP. Reitoria. Coordenadoria Geral de Bibliotecas, 1999. (Publicações Técnicas, 1).

Carneiro, M. V. (1985) Diretrizes para uma política de indexação. // Revista da Escola de Biblioteconomia da UFMG. Belo Horizonte. 14:2 (set. 1985) 221-241.

Fattahi, R. (1998) Library cataloguing and abstracting and indexing services: reconciliation of principles in the online environment? // Library Review. Bradford. 47:4 (1998) 211-216.

Fujita, M. S. L. (2005) Aspectos evolutivos das bibliotecas universitárias em ambiente digital na perspectiva da Rede de Bibliotecas da UNESP. // Informação \& Sociedade: estudos. João Pessoa. 15:2 (2005). http://www. informacaoesociedade.ufpb.br/pdf/IS1520504.pdf (22 mar. 2006).

Fujita, M. S. L. (1999) Leitura em análise documentária. Marília: Unesp; CNPq, 1999. Relatório parcial de pesquisa.

Fujita, M. S. L. (1999) A leitura do indexador: estudo de observação. // Perspectivas em Ciência da Informação. 4:1 (1999) 101-116.

Fujita, M. S. L. (2003) A leitura documentária do indexador: aspectos cognitivos e lingüísticos influentes na formação do leitor profissional. $321 \mathrm{f}$. Tese (Livre-Docência nas disciplinas Análise Documentária e Linguagens Documentárias Alfabéticas) - Faculdade de Filosofia e Ciência, UNESP.

Fujita, M. S. L.; Nardi, M. I. A.; Fagundes, S. A. (2003) Observing documentary reading by verbal protocol. // Information Research, 8:4 (2003) paper 155. http://in formationr.net/ir/8-4/paper155.html (10 jun. 2006).

Gil Leiva, I. (1999) Automatización de la indización de documentos. Gijón: Trea, 1999.

Gil Leiva, I. (2008) Manual de indización. Teoría y práctica. Gijón: Trea, 2008.

Mmartinelli, A. T. S. (1998) A base de dados bibliográficos de acervo como suporte para o processo de automação: uma experiência na UNESP - Universidade Estadual Paulista. // Seminário nacional de bibliotecas universitárias, 10., Fortaleza, 1998. Anais... Fortaleza: FINEP, 1998. (Publicado em disquete).

Rubi, M. P. (2000) Os serviços de análise e sua política de indexação. $80 \mathrm{f}$. Trabalho de Conclusão de Curso (Graduação em Biblioteconomia) - Faculdade de Filosofia e Ciências, Universidade Estadual Paulista; CNPq, Marília, 2000. 
UNESP. Reitoria. Coordenadoria Geral de Bibliotecas. (1998) Conversão retrospectiva de registros bibliográficos utilizando bases de dados em Cd-Rom. São Paulo, 1998. 7p. (Projeto apresentado à VUNESP).

\section{Apéndice: Questionário para entrevistas}

\begin{tabular}{|l|l|}
\hline Biblioteca/Arquivo & \\
\hline País & \\
\hline Director/a & \\
\hline Datos contacto & \\
\hline Fecha & \\
\hline
\end{tabular}

\section{Datos generales}

Fundación:

Fondos principales:

Personal y formación:

Proyectos en marcha (implantación de software, digitalización, etc.):

\section{Procedimiento}

¿Realizan la indización / asignación de los documentos o capturan los descriptores / materias de algún catálogo o base de datos?

No ( ) Relatar motivos, problemas e restrições

$\mathrm{Si}$

Indización / asignación propia

Captura de la indización

¿De dónde?

¿Disponen de un manual de procedimiento para la indización / asignación?

Sí

Comentar. ¿Está publicado, es público? No está publicado pero está disponible para los catalogadores en la red interna

No

¿Está establecido el grado de especificidad en la indización / asignación?

¿Hay indicaciones sobre el número de términos / materias por documento?

¿Hay indicaciones sobre el tiempo dedicado a este proceso?

¿Siguen alguna norma nacional o internacional sobre indización?

Todo esto está contenido en el documento.

¿Durante el proceso de indización / asignación se emplea alguna ayuda automática para facilitar esta operación?

Sí

Enumerar y describir

No
¿Utilizan algún sistema de validación automática de términos / materias para garantizar la coherencia en el catálogo / base de datos?

Sí

Describir : Listado de autoridades tanto de temas como de nombres (geográficos, onomásticos, identificadores, series y títulos)

No

¿Utilizan términos / materias no controlados, esto es, en lenguaje natural (¿Marc21 653?)?

\section{Lenguajes controlados}

Lenguajes de indización utilizados. Describir y enumerar ¿Participa actualmente o ha participado en proyectos de compatibilidad 0 interoperabilidad entre vocabularios controlados?

Sí

Enumerar y describir:

No

\section{Evaluación}

¿Realizan algún tipo de pruebas-ensayos para la evaluación periódica de la indización / asignación de materias?

Sí

¿De qué tipo? ¿Evaluación intra e inter indizadores / catalogadores?

¿Disponen de algún informe publicado o público?

No

\section{Política de indización}

¿Dispone la institución de una política de indización reglamentada?

Sí

¿Está publicada, es pública?

No

\section{Formación}

Número de profesionales dedicados a tareas de indización / asignación de materias

¿Los indizadores / catalogadores reciben cursos específicos sobre indización / asignación de materias cuando comienzan con esa tarea?

Sí

Tipo, duración, etc.:

No

¿Los indizadores / catalogadores reciben cursos de formación continua?

Sí

Tipo, duración, etc.:

No

Observaciones: 